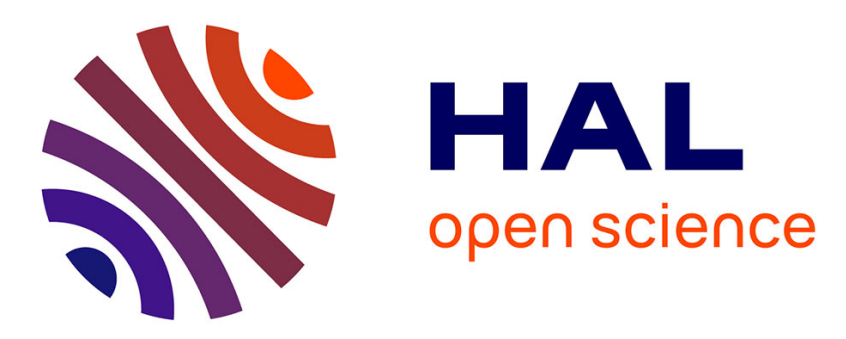

\title{
Two-phase damage theory and crustal rock failure: The theoretical 'void' limit, and the prediction of experimental data
}

Y. Ricard, D. Bercovici

\section{- To cite this version:}

Y. Ricard, D. Bercovici. Two-phase damage theory and crustal rock failure: The theoretical 'void' limit, and the prediction of experimental data. Geophysical Journal International, 2003, 155 (3), pp.1057-1064. 10.1111/j.1365-246X.2003.02112.x . hal-02046731

\section{HAL Id: hal-02046731 \\ https://hal.science/hal-02046731}

Submitted on 28 Jan 2021

HAL is a multi-disciplinary open access archive for the deposit and dissemination of scientific research documents, whether they are published or not. The documents may come from teaching and research institutions in France or abroad, or from public or private research centers.
L'archive ouverte pluridisciplinaire HAL, est destinée au dépôt et à la diffusion de documents scientifiques de niveau recherche, publiés ou non, émanant des établissements d'enseignement et de recherche français ou étrangers, des laboratoires publics ou privés. 


\title{
Two-phase damage theory and crustal rock failure: the theoretical 'void' limit, and the prediction of experimental data
}

\author{
Yanick Ricard ${ }^{1}$ and David Bercovici ${ }^{2}$ \\ ${ }^{1}$ Laboratoire des Sciences de la Terre, Ecole Normale Supérieure de Lyon, CNRS UMR5570, 46 allée d'Italie, F-69364 Lyon, Cedex 07, France. E-mail: \\ ricard@ens-lyon.fr \\ ${ }^{2}$ Department of Geology and Geophysics, Yale University, PO Box 208109, New Haven, CT 06520-8109, USA.E-mail:david.bercovici@yale.edu
}

Accepted 2003 August 22. Received 2003 July 28; in original form 2002 November 29

\begin{abstract}
S UMMAR Y
Using a classical averaging approach, we derive a two-phase theory to describe the deformation of a porous material made of a matrix containing voids. The presence and evolution of surface energy at the interface between the solid matrix and voids is taken into account with non-equilibrium thermodynamic considerations that allow storage of deformational work as surface energy on growing or newly created voids. This treatment leads to a simple description of isotropic damage that can be applied to low-cohesion media such as sandstone. In particular, the theory yields two possible solutions wherein samples can either 'break' by shear localization with dilation (i.e. void creation), or undergo shear-enhanced compaction (void collapse facilitated by deviatoric stress). For a given deviatoric stress and confining pressure, the dominant solution is that with the largest absolute value of the dilation rate, $|\Gamma|$, which thus predicts that shear-localization and dilation occur at low effective pressures, while shear-enhanced compaction occurs at larger effective pressure. Stress trajectories of constant $|\Gamma|$ represent potential failure envelopes that are ogive- (Gothic-arch-) shaped curves, wherein the ascending branch represents failure by dilation and shear-localization, and the descending branch denotes shear-enhanced compactive failure. The theory further predicts that the onset of dilation preceding shear-localization and failure necessarily occurs at the transition from compactive to dilational states and thus along a line connecting the peaks of constant- $|\Gamma|$ ogives. Finally, the theory implies that while shear-enhanced compaction first occurs with increasing deviatoric stress (at large effective pressure), dilation will occur at higher deviatoric stresses. All of these predictions in fact compare very successfully with various experimental data. Indeed, the theory leads to a normalization where all the data of failure envelopes and dilation thresholds collapse to a single curve.
\end{abstract}

Key words: fracture, damage, sandstone.

\section{INTRODUCTION}

Understanding how tectonic plates are spontaneously generated and how they interact with mantle convection is a major challenge in geodynamics. We know that some of the necessary ingredients of a plate rheology are a strain-rate weakening, a temperature dependence, a memory and a long-term healing capacity (see Bercovici et al. 2000, for a review). All of these ingredients can be found in theories where the rheology is a function of a damage parameter (or damage state variable) that obeys is own evolution equation.

In a series of papers, Bercovici et al. (2001a,b) (which we shall refer to herein as BRS1 and BRS2), Ricard et al. (2001) and Bercovici \& Ricard (2003) demonstrated a fruitful analogy between damage mechanics and two-phase fluid dynamics. In particular, the creation of cracks and voids involves the deposition of crack-surface energy in a way similar to the growth of surface energy on the interface between two immiscible phases. In BRS2, this analogy lead to a two-phase damage theory that was developed using two incompressible fluids. In this paper we will show that the theory can be further simplified when the less viscous phase is simply identified with vacuum or void, i.e. with a fluid without density, viscosity or pressure. We will then show that the properties of our two-phase void-matrix medium are indeed similar to those observed in laboratory experiments on rock deformation.

\section{BASIC THEORY}

We consider that a porous material (e.g. sandstone) is a mixture of matrix (e.g. silicic grains) and voids. It contains a quantity of voids which have a volume fraction $\phi$. The quantity $1-\phi$ is the fraction of pure matrix in a given volume of mixture, $\delta V$. As with all continuum models of two-phase flow, $\delta V$ is assumed to be larger than the volume of pores but small enough to resolve 'macroscopic' 
(in contrast to 'microscopic', which in two-phase media refers to the grain- and/or pore-scale) gradients in the medium. The porosity $\phi$ is therefore considered a continuous, well-behaved mathematical function (e.g. Drew 1971). A continuum description of the mixture can then be obtained by averaging the microscopic properties over the test volume $\delta V$. The macroscopic averages of the matrix true velocity, pressure and stresses over the complex topology of the pure matrix phase will be labelled $\mathbf{v}_{m}, P_{m}$ and $\tau_{m}$, respectively.

We consider that in the mixture, the matrix is isotropically connected. For simplicity, we define the matrix density $\rho_{m}$ and viscosity $\mu_{m}$ to be constants. The pure matrix material obeys the usual incompressible Navier-Stokes equations with true, microscopic velocities. The complexities of the macroscopic rheological behaviour of the two-phase mixture only come from the interplay between matrix and voids. Our approach is akin to rate and state models of localization (Sleep 2002), where the state variable is identified with porosity. The theory, however, could be generalized to cases where $\mu_{m}$ is variable or stress dependent.

The procedures to average the equations of mass, momentum and energy conservations in a mixture of matrix and void are in many aspects similar to those needed for a mixture of two fluids (see BRS1). They will not be repeated here in detail, we will simply emphasize the differences due to replacing a fluid of low viscosity by voids. The major ones are of course that there are no mass and momentum conservation equations for voids.

\subsection{Mass}

The equation of matrix conservation appears as

$\frac{\partial(1-\phi)}{\partial t}+\nabla \cdot\left[(1-\phi) \mathbf{v}_{m}\right]=0$.

This equation can be recast in terms of porosity advection and creation, i.e.

$\frac{D_{m} \phi}{D t}=\Gamma$,

where the Lagrangian derivative is

$\frac{D_{m}}{D t}=\frac{\partial}{\partial t}+\mathbf{v}_{m} \cdot \nabla$

in which the rate of matrix dilation, or void production, is

$\Gamma=(1-\phi) \nabla \cdot \mathbf{v}_{m}$

\subsection{Momentum}

The matrix force balance is identical to the total force balance equation (see eq. 76 of BRS1)

$$
\begin{aligned}
0= & -\nabla\left[(1-\phi) P_{m}\right]+\nabla \cdot\left[(1-\phi) \tau_{m}\right] \\
& -(1-\phi) \rho_{m} g \hat{z}+\nabla(\gamma \alpha) .
\end{aligned}
$$

The last term of this equation introduces $\gamma$, the surface tension, and $\alpha$, the interfacial area per unit volume of mixture. A gradient in porosity is associated with a gradient in surface tension that enters the momentum equation like an extra body force (just like the gradients of pressure or of viscous stresses). By grouping the three terms that contain the $\nabla$ operator, one sees that the macroscopic total stress in the porous material is

$\left.\Sigma=(1-\phi)\left(\boldsymbol{\tau}_{m}-P_{m} \boldsymbol{I}\right)+\gamma \alpha \boldsymbol{I}\right)$, where $\boldsymbol{I}$ is the identity tensor. In addition to the usual deviatoric and pressure terms, acting on a proportion $1-\phi$ of matrix, $\gamma \alpha$ is the effective contribution to pressure by interface surface tension.

The force balance eq. (5) is not identical to that derived in BRS1 (eq. 73) for a two-phase flow. In the case where the voids are filled with a fluid, some shear stresses can be transmitted through interfaces and modelled by a Darcy interaction term, and surface tension can be balanced not only by matrix stresses but also by fluid stresses (see Bercovici \& Ricard 2003, for more details).

As in BRS1, matrix stresses are simply given by

$\boldsymbol{\tau}_{m}=\mu_{m}\left[\nabla \mathbf{v}_{m}+\left[\nabla \mathbf{v}_{m}\right]^{t}-\frac{2}{3}\left(\nabla \cdot \mathbf{v}_{m}\right) \boldsymbol{I}\right]$,

i.e. although the macroscopic matrix flow looks compressible, no bulk viscosity explicitly enters the rheological equation relating macroscopic stresses and velocities.

\subsection{Energy}

Similarly to what has been found in BRS1 (eqs 78 and 79) but assuming that interfacial energy is transported only with the matrix, we arrive at the entropy equation

$$
\begin{aligned}
& (1-\phi) \rho_{m} c_{m} \frac{D_{m} T}{D t}-T\left[\frac{D_{m}}{D t}\left(\alpha \frac{d \gamma}{d T}\right)+\alpha \frac{d \gamma}{d T} \nabla \cdot \mathbf{v}_{m}\right] \\
& =Q-\nabla \cdot \mathbf{q}+(1-f) \Psi+B\left(\frac{D_{m}}{D t} \phi\right)^{2}
\end{aligned}
$$

(see also Bercovici \& Ricard 2003). The term $-\alpha(d \gamma / d T)$ represents interfacial entropy and $\alpha(d \gamma / d T) \nabla \cdot \mathbf{v}_{m}$ is similar to an adiabatic cooling term (i.e. dilating the mixture and increasing the interface area while holding the interface entropy constant, induces an effective loss of interface entropy per unit area to compensate). The term $\Psi$ is positive definite and represents the deformational work

$\Psi=(1-\phi) \nabla \mathbf{v}_{m}: \tau_{m}$.

Isotropic viscous compaction introduces another positive entropy source $B\left(D_{m} \phi / D t\right)^{2}$, where the parameter $B$ will be discussed in the next paragraph (see also BRS1).

However, for a two-phase flow it has been argued in BRS1 that only a quantity $1-f$ of the deformational work $(0 \leq f \leq 1)$ is transformed into heat production. A proportion $f$ goes rather into making new interfaces. This leads to the damage equation

$$
\left(P_{m}+\gamma \frac{d \alpha}{d \phi}\right) \frac{D_{m} \phi}{D t}=-B\left(\frac{D_{m} \phi}{D t}\right)^{2}+f \Psi .
$$

This equation is related to the microscopic stress-continuity boundary conditions at the matrix-void interfaces. In the following, using eq. (2), we will make use of the damage equation on the form

$f \Psi=\left(P_{m}+\gamma \frac{d \alpha}{d \phi}+B \Gamma\right) \Gamma$.

In BRS1, it was shown that in the case of spherical empty bubbles of radius $R$, the term $d \alpha / d \phi$ is simply the total curvature $2 / R$, which enters the classical Laplace equilibrium surface-tension condition, which for our medium would appear as $P_{m}=-2 \gamma / R$ since the voids exert no pressure. Therefore, near equilibrium the right-hand side of eq. (11) is zero and the Laplace condition is obtained. At low energy input (i.e. $\Psi=0$ ) the departure from the Laplace condition is proportional to the rate of void formation: a matrix pressure larger 
than the Laplace condition closes the voids. A high energy input (i.e. $\Psi \neq 0$ ) can favour the formation of new cracks.

The three scalar eqs (2), (8) and (11) and the vectorial equation (5), control the behaviour of the mixture defined by the six variables $\phi, \mathbf{v}_{m}, P_{m}$ and $T$. These equations must be augmented by three phenomenological equations; the rheological law eq. (7), the empirical relationship relating the interfacial area density $\alpha$ to the porosity $\phi$ (Ni \& Beckerman 1991; Bercovici et al. 2001a) and the expression for $B$. A complete discussion of the differences between the various two-phase formalisms, that of McKenzie (1984), that of BRS1 and this void-matrix limit is presented in Bercovici \& Ricard (2003).

\subsection{Damage equation in terms of principal stresses}

As shown in BRS1, the coefficient $B$ controls the resistance of the mixture to isotropic deformation. Even when the matrix is isotropically deformed at macroscopic scale, there are still deviatoric stresses at the microscopic or grain scale. The average of these microscopic viscous stresses is equivalent to a finite macroscopic isotropic stress or pressure (see BRS1). In the simplest cases of a purely viscous, connected matrix, $B$ has the form

$B=\frac{K_{0} \mu_{m}}{\phi(1-\phi)}$,

where the coefficient $K_{0}$ can be computed analytically for pores of simple topology (e.g. spheres and cylinders) and is always close to 1 . The coefficient $B$ plays a role similar to that of the bulk viscosity in the two-phase equations of McKenzie (1984).

Next, we apply the damage equation, eq. (11), to porous rocks. Porous rocks have intrinsically different behaviours under tension and compression. Under compression, we assume that the micromechanical model that we developed for a viscous matrix still applies such that $K_{0} \sim 1$. Under tension, however, low-cohesion porous materials undergo deformation and failure at much smaller tensile stresses and with much less resistance than when under compression. The grain separation can occur without intrinsic deformation of each grain. We therefore postulate that $K_{0} \ll 1$ under tension.

To make our theoretical results comparable with laboratory observations, we now rewrite the damage equation, eq. (11), in terms of principal stresses. We define $\sigma_{i}, i=1,2,3$ as the three principal total stresses externally applied during an experiment and adopt the convention that compactive stresses are positive. The local integration of the force balance equation, as in eq. (6) indicates that

$(1-\phi)\left(\tau_{m i}-P_{m}\right)+\gamma \alpha=-\sigma_{i}$

where $\tau_{m i}$ is the deviatoric matrix normal stress along the principal axis associated with $\sigma_{i}$; for simplicity, gravitational effects are neglected, or cancelled with hydrostatic pressure gradients.

After some algebraic manipulation, the matrix pressure, the deviatoric stresses and the deformational work can be expressed in terms of the principal stresses $\sigma_{i}$, which leads to

$P_{m}=\frac{1}{(1-\phi)}(\gamma \alpha+P)$,

$\Psi=\frac{1}{1-\phi} \frac{Q^{2}}{3 \mu_{m}}$,

where the mean stress $P$, and the rms differential stress $Q$ (which are related to the first two stress invariants) are

$P=\frac{\sigma_{1}+\sigma_{2}+\sigma_{3}}{3}$,
$Q^{2}=\frac{3}{2}\left[\left(\sigma_{1}-P\right)^{2}+\left(\sigma_{2}-P\right)^{2}+\left(\sigma_{3}-P\right)^{2}\right]$.

$Q$ has been defined according to Wong et al. (1997) such that under uniaxial compression $\left(\sigma_{2}=\sigma_{3}\right), Q$ is simply $\left|\sigma_{1}-\sigma_{3}\right|$. Under simple shear $\left(\sigma_{2}=-\sigma_{1}, \sigma_{3}=0\right), Q$ is $\sqrt{3}\left|\sigma_{1}\right|$.

Taking into account our assumption that $K_{0}(\Gamma)$ is either approximately 0 or $K_{0}$, we can rewrite the damage equation (11) in terms of the effective and differential stresses $P$ and $Q$ [using eqs (14) and (15)]

$\left(\frac{Q}{Q^{*}}\right)^{2}=\frac{P+P_{\gamma}}{P^{*}} \frac{\Gamma}{\Gamma_{0}} \quad$ if $\quad \Gamma>0$,

$\left(\frac{Q}{Q^{*}}\right)^{2}=\frac{1}{P^{*}}\left(P+P_{\gamma}+P^{*} \frac{\Gamma}{\Gamma_{0}}\right) \frac{\Gamma}{\Gamma_{0}} \quad$ if $\quad \Gamma<0$,

where we introduce the new quantities, $P_{\gamma}, P^{*}, Q^{*}$ given by

$P_{\gamma}=\gamma\left[\alpha+(1-\phi) \frac{d \alpha}{d \phi}\right]$

$P^{*}=\frac{K_{0}}{\phi} \mu_{m} \Gamma_{0}$,

$Q^{*}=\sqrt{\frac{3 K_{0}}{\phi f}} \mu_{m} \Gamma_{0}$.

The quantity $\Gamma_{0}$ is an arbitrary rate of porosity change. As demonstrated in Ricard et al. (2001), for a very general choice of physically admissible $\alpha(\phi)$ relationships, $P_{\gamma}$ is positive for all $\phi$.

The $P-Q$ trajectories of eqs (18) and (19) corresponding to constant $\Gamma$ are depicted in Fig. 1. For positive $\Gamma$ (i.e. material dilation), eq. (18) predicts that $Q$ is proportional to $\sqrt{P+P_{\gamma}}$. If, in eq. (18), the differential stress $Q$ is held at a finite value while the mean compressive stress $P$ approaches $-P_{\gamma}$ from positive values, then $\Gamma$ necessarily goes to $+\infty$. Physically this means that once a negative mean stress $P$ cancels out the effective cohesion provided by surface

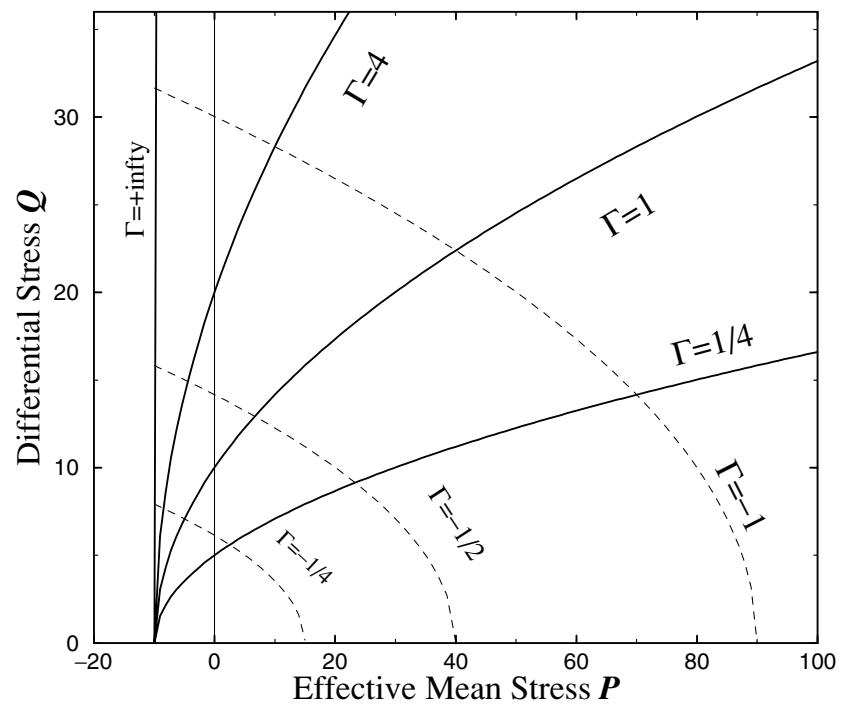

Figure 1. Constant rates of void production in the $P-Q$ space according to eqs (18) and (19). We arbitrarily use $P^{*}=90, Q^{*}=30, P_{\gamma}=10, \Gamma_{0}=1$ and various $\Gamma$ that are indicated along the curves (these values correspond to $K_{0}=1, \mu=0.9, f=0.27, \phi=0.01$ ). The constant rates of expansion, $\Gamma>0$ or compaction, $\Gamma<0$, correspond to parabolas. When the effective mean stress overcomes the cohesion pressure $\left(P<-P_{\gamma}\right)$ an instantaneous rupture is predicted $(\Gamma=+\infty)$. 
tension (which acts to collapse voids and to compact the material), then any differential stress $Q$ in excess of the mean stress will cause instantaneous damage and dilation of the matrix. Thus, no finite solutions are allowed for even more negative mean stresses (i.e. $P<-P_{\gamma}$ ). Realistically, of course, there should be some small but finite resistance to dilation rather than, as we assume, $K_{0}=0$ for $\Gamma$ $>0$, which would thus prohibit such explosive behaviour. What is important to emphasize, however, is that $P_{\gamma}$ is therefore the effective cohesive strength of the material. However, surface tension $\gamma$ of rocks is a relatively small number (typically $1 \mathrm{Jm}^{-2}$ ) and $P_{\gamma}$ is likewise small $\left(P_{\gamma} \ll P^{*}\right)$, which corresponds well to low-cohesion materials such as sandstone.

The solutions for compaction $(\Gamma<0)$ also correspond to parabolas in the $P-Q$ space. The rate of porosity reduction $\Gamma$ goes as $-(P$ $\left.+P_{\gamma}\right) \Gamma_{0} / P^{*}$ at large effective mean stress, and as $-\left(Q / Q^{*}\right) \Gamma_{0}$ at large differential stress. Note that in the absence of imposed stresses, $P=Q=0$, the surface tension term will slowly close the porosity at the rate $\Gamma=-\Gamma_{0} P_{\gamma} / P^{*}$. This self-healing of the porous material, converts surface energy back into heat through the source term $B \Gamma^{2}$ (see eq. 8).

\subsection{Theoretical prediction}

Our theory predicts that under given $P$ and $Q$ conditions, the system will evolve by changing its porosity. However, two solutions are possible

$\Gamma^{+}=\Gamma_{0} \frac{P^{*}}{P+P_{\gamma}}\left(\frac{Q}{Q^{*}}\right)^{2}$,

$\Gamma^{-}=-\frac{\Gamma_{0}}{2}\left[\frac{P+P_{\gamma}}{P^{*}}+\sqrt{\left(\frac{P+P_{\gamma}}{P^{*}}\right)^{2}+4\left(\frac{Q}{Q^{*}}\right)^{2}}\right]$,

which are solution of eqs (18) and (19), respectively (we can neglect a third solution similar to eq. (24), but with a minus sign in front of the radical since this only permits $\Gamma^{-}>0$ ).

Any solution with non-zero $\Gamma$ is unstable (since it entails evolution away from the initial porosity $\phi$ ); however, the dominant solution, i.e. the most unstable, for the given stress state is necessarily that with largest absolute dilation rate $|\Gamma|$, i.e. the absolute dilation rate will be $\max \left(\left|\Gamma^{+}\right|,\left|\Gamma^{-}\right|\right)$. This solution is, in effect, the one that mitigates an unstable stress state most rapidly. Contours of $\max \left(\left|\Gamma^{+}\right|,\left|\Gamma^{-}\right|\right)$are shown in Fig. 2 and appear as ogive, or Gothic-arch-shaped curves. The branch of the curve to the left of the ogive peaks represents dilation and those to the right represent compaction.

We contend that empirical failure envelopes will effectively fall on these ogive curves because either 'shear localization' or 'cataclastic compaction' is detected experimentally when $|\Gamma|$ exceeds a threshold value, say $\Gamma_{0}$, i.e. when $\Gamma>\Gamma_{0}$, shear-localization is observed; when $\Gamma<-\Gamma_{0}$, compaction is observed. For example, the experimental detection for failure possibly occurs when the dilation or compaction rate exceeds the given adjustment rate for the loading apparatus (e.g. natural compaction that is faster than the compression rate of the loading apparatus will appear as the first measurable strength loss). Thus failure would be detected at or near a given dilation rate which is itself determined by machine properties. Therefore, we predict that the ogive-shaped contours of $\max \left(\left|\Gamma^{+}\right|,\left|\Gamma^{-}\right|\right)$ also represent possible failure envelopes.

The onset of dilation as a precursor to shear-localization failure will occur at the transition from compactive to dilational modes. A stress trajectory with a fixed value of $P$, but with $Q$ increasing from zero, will intersect the compressive branches of ogive curves

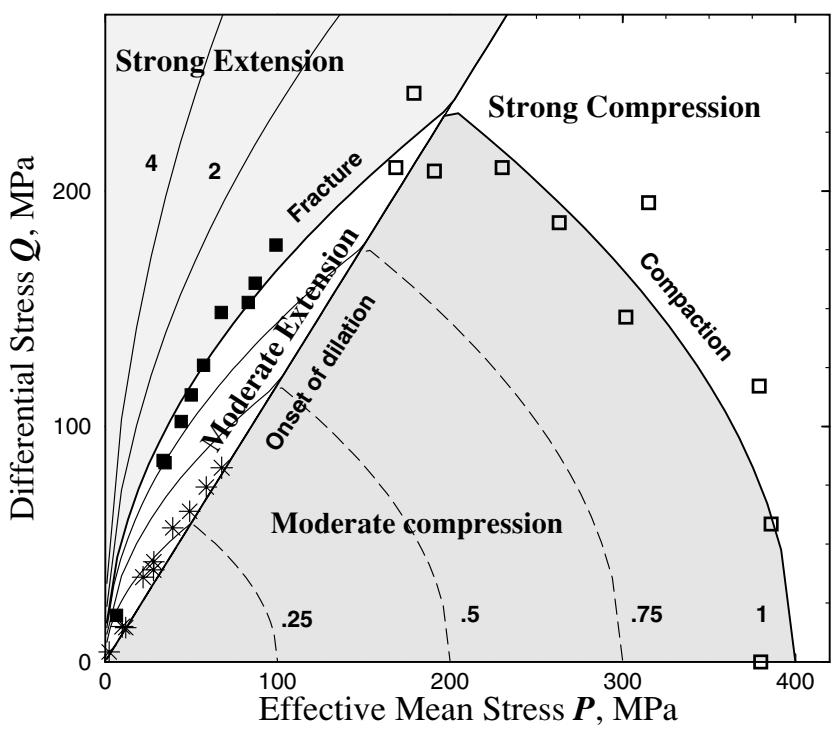

Figure 2. Experimental data on Berea sandstone as a function of effective mean stress and differential stress. Various symbols depict the onset of shearinduced dilation (stars), the fracture (filled squares), the shear-enhanced compaction (open squares). The data are close along the solutions of eqs (18) and (19) corresponding to the largest absolute value of the porosity change. Before reaching the domain of strong extension (light grey), the system in moderate compression (dark grey) has first to cross the line of onset of dilation eq. (25). The solid lines correspond to extension, the dashed lines to compression. They correspond to $\left|\Gamma / \Gamma_{0}\right|=0.25,0.5,0.75,1,2$ and 4 where $\Gamma_{0}$ corresponds to the level of porosity change on the observed failure envelope.

until it intersects the peak of an ogive curve, after which it will intersect dilational branches (see Fig. 2). Thus the onset of dilation (transition from compactive to dilational) for any arbitrary $P$ will coincide with the peak of an ogive curve, which occurs where $\Gamma^{+}$ $=\left|\Gamma^{-}\right|$. The onset curve is therefore the line connecting all ogive peaks, as shown in Fig. 2.

The $P-Q$ space is therefore divided into two domains, one of compression, one of dilation separated by the dilation onset curve $\Gamma^{+}=-\Gamma^{-}$, which is simply the line

$Q=v\left(P+P_{\gamma}\right)$,

where

$v=\sqrt{2} \frac{Q^{*}}{P^{*}}=\sqrt{\frac{6 \phi}{K_{0} f}}$.

This linear relationship looks like a generalization of the MohrCoulomb criterion (or Amonton's law) where $v$ and $v P_{\gamma}$ are somewhat equivalent to a coefficient of internal friction and a critical yield stress (Jaeger \& Cook 1979). However, there are two major differences. Eq. (25) gives the onset of dilation, not the failure criterion and the intermediate stress $\sigma_{2}$ enters in $P$ while the Mohr-Coulomb criterion is strictly bidimensional.

Below the line given by eq. (25) the system is in compression and above it is in expansion. The base of the ogive of constant $|\Gamma|$ in $P-Q$ space (i.e. where it intersects the line $Q=0$ ) spans between $P=-P_{\gamma}$ and $P=P^{*}|\Gamma| / \Gamma_{0}-P_{\gamma}$; the summit of these ogives are at $P=\frac{1}{2} P^{*}|\Gamma| / \Gamma_{0}-P_{\gamma}$ and $Q=Q^{*}\left(|\Gamma| / \Gamma_{0}\right) / \sqrt{2}$. These different curves and domains illustrated in Fig. 2 correspond topologically to the schematic map proposed in Zhu \& Wong (1997).

For $0<P+P_{\gamma}<P^{*} / 2$ and small $Q$, (in the dark-grey zone of Fig. 2 and left of the ogive summit) the system is always mildly 
compacting $\left(-\Gamma_{0}<\Gamma<0\right)$. When $Q$ increases, the system remains in this mild compactive regime, then it crosses the onset of dilation $\left(\Gamma^{+}=\left|\Gamma^{-}\right|\right)$and enters a mild disruptive regime where $0<\Gamma<\Gamma_{0}$ (white zone in Fig. 2 labelled 'moderate extension'). Eventually it breaks into the light-grey zone $\left(\Gamma>\Gamma_{0}\right)$.

The situation for $P+P_{\gamma}>P^{*} / 2$ (right of the ogive summit) is different. When $Q$ increases from zero, the sample crosses the compactive branch, from dark-grey to white $\left(-\Gamma^{-}=\Gamma_{0}\right)$, but eventually should cross the dilation branch (from white to light-grey) $\left(\Gamma^{+}=\Gamma^{-}\right)$. In other words, when increasing the shear stress, a sample that breaks will not compact at larger shear stress but a sample that undergoes cataclastic compaction, should break at a larger shear stress.

In summary, the major predictions of the theory are: (1) failure envelopes for either dilational shear-localization, or shearenhanced compaction will occur on ogive-shaped curves of constant $\max \left(\left|\Gamma^{+}\right|,\left|\Gamma^{-}\right|\right)$; (2) the onset of dilation will occur along a line connecting the peaks of these ogive curves, i.e. where $\Gamma^{+}=\left|\Gamma^{-}\right|$; and (3) at high confining pressure $P$ shear-enhanced compactive failure will initially ensue with increasing shear stress $Q$, but dilation and shear-localization can occur at much higher $Q$. We will see that these predictions match a surprisingly wide array of experimental data.

\section{COMPARISON WITH EXPERIMENTS}

It may seem strange to use viscous modelling to discuss the mechanical deformation of a porous material where the conventional approach is to use an elastic rheology with failure (Fung 1977) or damage (Lyakhovsky et al. 1997). However, fracture and compaction are irreversible processes that are probably as much related to viscosity as to elasticity. In fact, in this paper, the viscosity is always multiplied by $\Gamma_{0}$ and their product which has a dimension of stress simply enters as a measure of the resistance of the material to failure or cataclastic compaction.

Laboratory measurements on mechanical deformation of porous rocks have been performed by various authors. In this paper, we use experiments mostly on sandstones (Schock et al. 1973; Jamison \& Teufel 1979; Khan et al. 1991; Wong et al. 1997), except for one on limestone (Baud et al. 2000). The authors use triaxial experiments to investigate the non-elastic properties of the natural materials either under brittle failure or under cataclastic compaction. They observe that the failure envelopes are both functions of the effective mean stress $P$ and of the differential stress $Q$ (in their experiments the pore pressure $P_{\mathrm{p}}$ is non-zero, but once they decrease the mean stress by $P_{\mathrm{p}}$, their $P$ and $Q$ variables are identical to ours).

Experimental failure data from various experiments on Berea sandstones (Jamison \& Teufel 1979; Khan et al. 1991; Wong et al. (1997) are plotted in Fig. 2 along with our theoretical curves; it is immediately clear that the empirical failure envelope coincides with an ogive of constant $|\Gamma|$ (where the brittle fracture are depicted by filled squares, the shear-induced compaction by open squares). To scale the curves to the experimental data we have chosen for our theory $P_{\gamma} \sim 0$ (the cohesion is experimentally very low for sandstones), $P^{*}=400 \mathrm{MPa}, Q^{*}=334 \mathrm{MPa}$ and $|\Gamma|=\Gamma_{0}=1$ on the failure envelope. The data on onset of dilation and shear-induced dilation (stars) is also shown and lies very close to the line joining the summits of the various ogive curves corresponding to different $|\Gamma|$, as predicted.

Since the failure envelopes appear to fall on curves of constant $|\Gamma|$, we can set the arbitrary scale $\Gamma_{0}$ to this constant and thus choose $|\Gamma| / \Gamma_{0}=1$ on the failure envelope and rewrite the damage equations

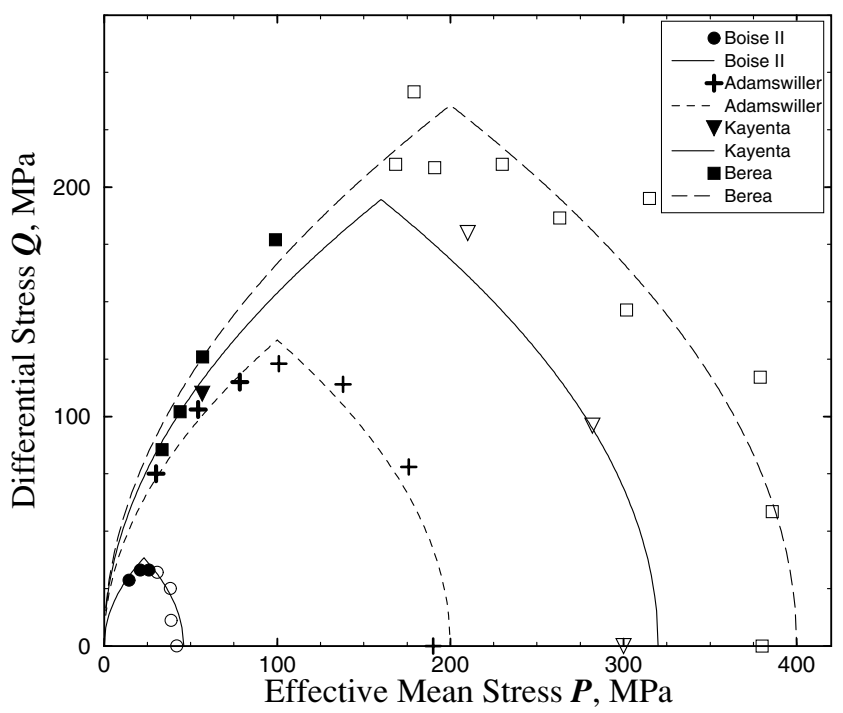

Figure 3. Theoretical failure envelopes compared with laboratory experiments performed on various sandstone samples (Wong et al. 1997; Jamison $\&$ Teufel 1979). According to eqs (27) and (28), each envelope has a maximum at $Q=Q^{*}$ that separates the domains of shear localization failure (at low $P$, depicted with filled symbols) and of the shear enhanced compaction (at larger $P$, depicted with empty symbols). Without differential stress, the sample crushes under the hydrostatic pressure $P^{*}$. The values of $P^{*}$ and $Q^{*}$ are summarized in Table 1.

eqs (18) and (19) as

$\left(\frac{Q}{Q^{*}}\right)^{2}=\frac{P+P_{\gamma}}{P^{*}} \quad$ under shear localization,

$\left(\frac{Q}{Q^{*}}\right)^{2}=\frac{P^{*}-P-P_{\gamma}}{P^{*}} \quad$ under cataclastic compaction.

The summit of the failure ogive is therefore at $Q^{*} / \sqrt{2}$ and this value corresponds to the maximum differential stress that can be sustained by a sample. The existence of a differential stress above which yield occurs (independently of the effective pressure) is usually called the von Mises (1913) criterion. The quantity $P^{*}-P_{\gamma}$ becomes the onset of grain crushing (the right foot of the shear-enhanced compaction curve at $Q=0$ ).

The above failure predictions are in remarkable agreement with all the experimental data that we collected. Fig. 3 depicts the observations of rock failure (filled symbols) and cataclastic compaction (empty symbols) for three sandstone samples (Boise II, Adamswiller, Kayenta) (Wong et al. 1997). For Berea sandstone we reported the experiments already plotted in Fig. 2. The $P^{*}, Q^{*}$ values that we use to draw the theoretical curves are summarized in Table 1. For these experiments we are not able to distinguish $P_{\gamma}$ from 0 , although the cohesion strength cannot be zero. This agrees with the findings of Wong et al. (1997) where the authors had assumed that $P_{\gamma}=2$ per cent $P^{*}$. Table 1 includes the parameters needed to fit to other sandstones (Rothbach, Darley Dale and Lance) and a limestone (Solnhofen) that are not plotted in Fig. 3 to avoid too much overlapping between curves.

In the case of shear localization and when $P_{\gamma}=0$, the failure criterion eq. (27) corresponds exactly to Murrel's extension of the Griffith criterion (Griffith 1921; Murrell 1963). This extension arises naturally from our theory although, traditionally it is obtained without proof as a generalization of the more special plane Giffith criterion. 
Table 1. Mechanical data fitted by eqs (27) and (28). We deduced these values from a fit over the whole failure envelope, whereas. The values of $P^{*}$ (second column) are within a few MPa different from those reported in table 4 of Wong et al. (1997) for his own data on effective grain crushing pressure without differential stress.

\begin{tabular}{lcccc}
\hline Material & $\begin{array}{c}P^{*} \\
(\mathrm{MPa})\end{array}$ & $\begin{array}{c}Q^{*} \\
(\mathrm{MPa})\end{array}$ & $\begin{array}{c}P_{\gamma} \\
(\mathrm{MPa})\end{array}$ & Porosity \\
\hline${ }^{1}$ Sandstone Boise II & 46 & 54 & 0 & 0.35 \\
${ }^{1}$ Sandstone Adamswiller & 200 & 188 & 0 & 0.23 \\
${ }^{1}$ Sandstone Rothbach1 & 220 & 185 & 0 & 0.20 \\
${ }^{1}$ Sandstone Kayenta & 320 & 276 & 0 & 0.21 \\
${ }^{1}$ Sandstone Darley Dale & 380 & 300 & 0 & 0.15 \\
${ }^{1,2,3}$ Sandstone Berea & 400 & 334 & 0 & 0.21 \\
${ }^{4}$ Sandstone Lance & 950 & 530 & 70 & 0.075 \\
${ }^{5}$ Limestone Solnhofen & 570 & 618 & 40 & 0.03 \\
\hline
\end{tabular}

${ }^{1}$ From Wong et al. (1997).

${ }^{2}$ From Khan et al. (1991).

${ }^{3}$ From Jamison \& Teufel (1979).

${ }^{4}$ From Schock et al. (1973) reinterpreted in 5.

${ }^{5}$ From Baud et al. (2000).

The presence of $P_{\gamma}$ in eq. (27) increases the sample resistance to failure. The uniaxial compressive strength, $C_{0}$, is often chosen as a measure of rock strength. Replacing $\sigma_{1}=C_{0}, \sigma_{2}=\sigma_{3}=0$ in eqs (16) and (17) implies that $P=C_{0} / 3$ and $Q=C_{0}$, then by solving eq. (27), one obtains

$C_{0}=\frac{C}{2}\left(1+\sqrt{1+12 \frac{P_{\gamma}}{C}}\right)$,

where

$C=\frac{Q^{* 2}}{3 P^{*}}=\frac{\mu_{m} \Gamma_{0}}{f}$.

The presence of surface energy through the equivalent pressure $P_{\gamma}$ increases the strength of the sample above the value $C_{0}=C$.

In the previous fits, summarized in Table 1 , we assumed that $P^{*}$ and $Q^{*}$ were independent, arbitrary parameters although they should verify eqs (21) and (22). Eq. (21) suggests a $K_{0} \mu_{m} \Gamma_{0} / \phi$ dependence of $P^{*}$. From Table 1, taking all the sandstone data together, we obtain a reasonable fit with

$P^{*}(\mathrm{MPa})=\frac{65}{\phi}$,

with a large standard deviation of $84 \mathrm{MPa}$ (see Fig. 4). This suggests that the viscosity is roughly equal for all these samples that have rather similar petrologies $(\sim 70$ per cent quartz, $\sim 15$ per cent feldspar and variable quantities of clay, mica, oxides, carbonates, etc.).

More likely, the rheology of each sample is different and eq. (21) should rather be used to estimate their viscosity $\mu_{m}$. From Fig. 4 , it is clear that a $\phi^{-n}$ dependence of $P^{*}$ with $n \sim 1.5$ would give a better fit. There is also a threshold in porosity near 37 per cent above which sandstones become completely cohesionless (Jizba 1991; Vernik et al. 1993). An estimate of $\mu_{m}$ as a function of porosity and grain size can be found in Zhang et al. (1990).

Using eq. (26) the theory also indicates that

$\left(\frac{P^{*}}{Q^{*}}\right)^{2}=\frac{K_{0} f}{3 \phi}$

where the parameter $K_{0}$ is close to 1 . Although we have never claimed that $f$ should be the same for each sample, we must at least check that the data does not contradict the requirement that

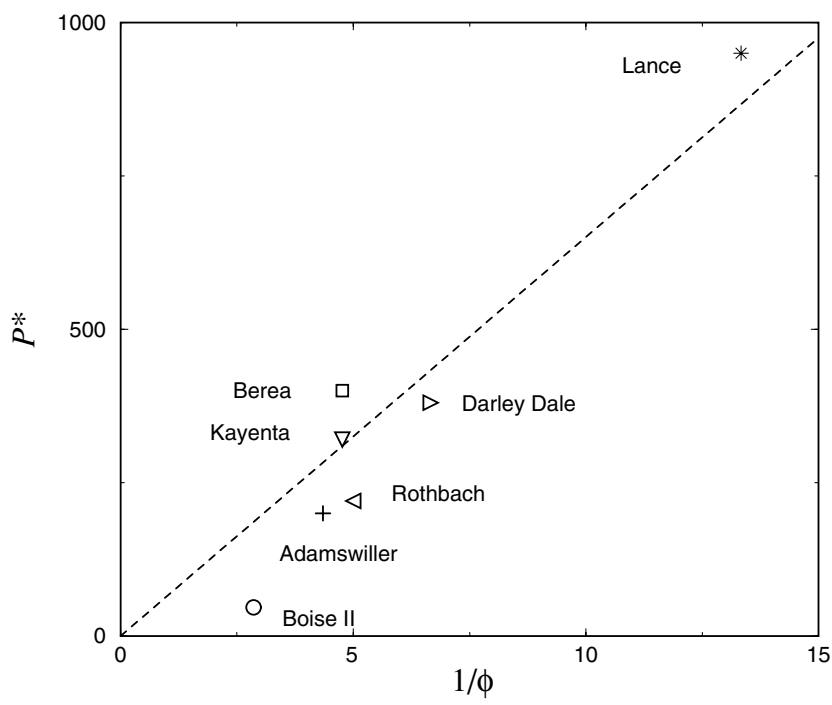

Figure 4. $P^{*}$ as a function of $1 / \phi$. The dotted line corresponds to a slope $K_{0} \mu_{m} \Gamma_{0}=65 \mathrm{MPa}$.

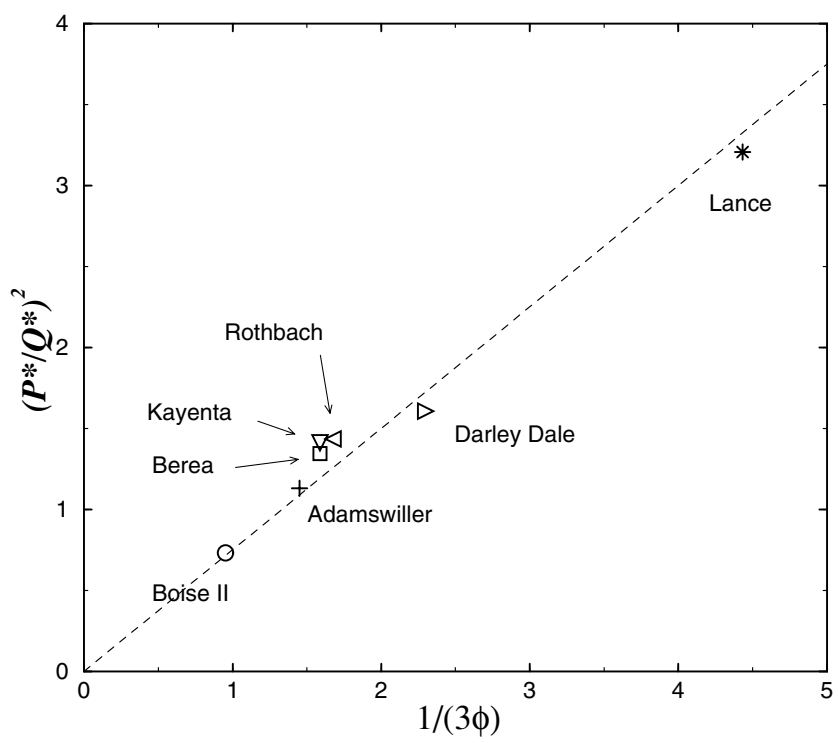

Figure 5. $\left(P^{*} / Q^{*}\right)^{2}$ should be proportional to $1 / \phi$ according to eq. (32) if the percentage $f$ of deformational work that can be stored in making new interfaces is independent of the samples. The experiments suggest that this is the case with $f \sim 75$ per cent for sandstones (dashed line).

$f \leq 1$. Using the sandstone data of Table 1, Fig. 5 depicts $\left(P^{*} / Q^{*}\right)^{2}$ as a function of $1 / \phi$. We verify that for each sample, the parameter $f$ is lower than 1 and more surprisingly that all sandstones seem to have the same $f$ parameter of order 0.75 (dashed line), assuming $K_{0}=1$. However, this value of $f$ corresponds to the occurrence of failure, i.e. at a specific dilation rate $\Gamma=\left|\Gamma_{0}\right|$; in general, $f$ is not independent of $\Gamma$ (see BRS2).

An independent lower bound for $f$ can also be obtained through eqs (29) and (30), which lead to

$\mu_{0} \Gamma_{0}=f C \leq f C_{0}$.

The relation eq. (31) indicates that $\mu_{0} \Gamma_{0} \sim 65 \mathrm{MPa}$, while a large compilation of sandstone compressive strengths (Vernik et al. 1993) gives $C_{0} \leq 254 \mathrm{MPa}$. These values imply $f \geq 0.25$, in agreement with our estimate of $f=0.75$. 
The data set from Baud et al. (2000) suggests that a much lower value of $f$ is appropriate for limestones. According to Table $1, f$ deduced from eq. (32) is only 0.075 . This implies that less deformational work goes into making new voids (and thus more work is dissipated) during deformation for limestones than for sandstones.

Assuming therefore that all samples have the same $K_{0}=1$ coefficients we conclude that all experiments follow the same failure envelope

$\tilde{Q}=\min (\sqrt{\tilde{P}}, \sqrt{1-\tilde{P}})$,

and the same onset of dilation

$\tilde{Q}=\sqrt{2} \tilde{P}$

where

$\tilde{P}=\frac{P+P_{\gamma}}{P^{*}}$

$\tilde{Q}=\frac{Q \sqrt{K_{0} f}}{P^{*} \sqrt{3 \phi}}$

Fig. 6 shows that the theoretical eq. (34) and the normalized data are in very good agreement. In this plot we use the same $f=0.75$ partitioning coefficient for all sandstones and $f=0.075$ for the limestone. A slightly better agreement could have been obtained by fitting all the $f$ s separately.

For two samples of lower porosity, Lance and Solnhofen, the cohesion pressure is non-zero (see Table 1). For these samples dilatant cataclastic failure have been observed for shear stresses above the onset of shear-induced compaction as predicted in Fig. 2. These observations are depicted in Fig. 6(b) and correspond to the few data above the ogive-type curve. They roughly prolongate the line corresponding to the onset of dilation. Two other experimental data for Lance sandstone at larger stresses have not been plotted for clarity but are in the predicted direction $((\tilde{P}, \tilde{Q})=(1.23,2.3)$ and $(1.55$, $2.87)$ ). Taking into account the fact that these samples went through shear-enhanced compaction before reaching a domain of extension, we think that the agreement between these difficult experiments and our simplistic theory is still surprisingly good.

On the right of the onset of the dilation-onset curve, away from the failure envelope, the rate of porosity change, $\Gamma$, should be given by eq. (24). On the left, the dilation should be given by eq. (23). However, it is quite difficult to compare these predictions of occurrences away from the failure envelope with experiments that mostly record the failure event. Moreover, it is quite probable that our assumption of purely Newtonian viscous matrix is too simplistic to give precise predictions over the whole $P-Q$ space.

\section{CONCLUSION}

We have briefly developed the void limit of the two-phase equations that were proposed in BRS1. We defer to a companion paper (Bercovici \& Ricard 2003) for a precise discussion of how the stresses and surface tension are distributed over the two phases, or only over the matrix phase when the fluid phase becomes rigorously inviscid.

Although our theory is based only on relatively simple first principles, it predicts failure envelope curves that include both dilational shear localization at low mean stress (confining pressure), and shearenhanced compaction at large mean stress, as well as the onset of dilation that is a precursor to shear localization. The theory further implies a failure criterion that generalizes the Murrell-Griffith rule
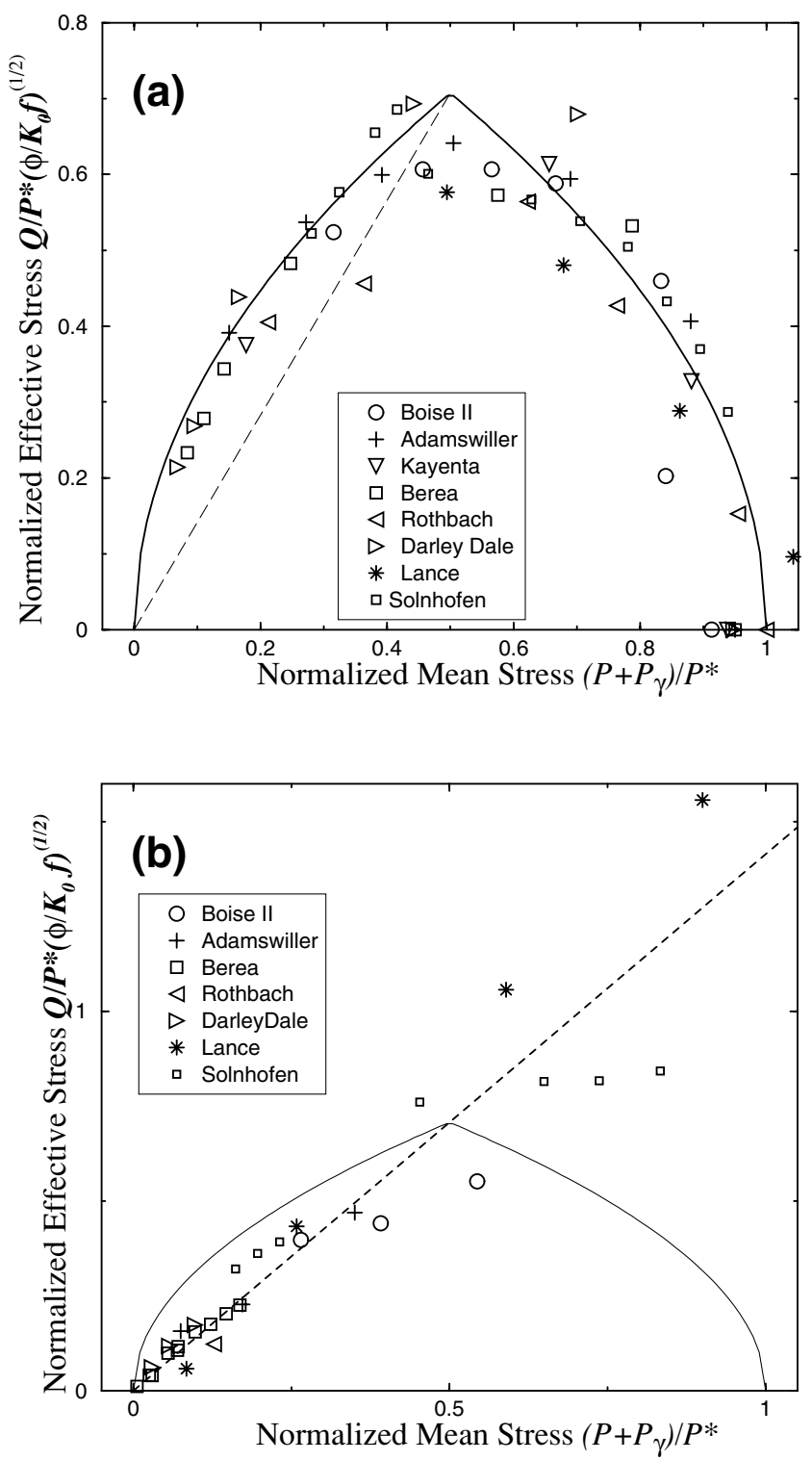

Figure 6. (a) Theoretical failure envelope (solid line) compared with various normalized experiments with seven sandstones and one limestone. (b) Theoretical onset of dilation (dashed line) compared with various normalized experiments. The few points above the ogive curve (two stars for Lance sandstone and four small squares for Solnhofen limestone) corresponds to dilatant cataclastic failure that have been observed for shear stresses above the onset of shear-induced compaction.

for shear-induced dilation. The same formalism also predicts that even after the occurrence of shear-enhanced compaction at moderate to low deviatoric stress (and high mean stress), dilation and shear localization can also occur once the deviatoric stress is increased sufficiently. In the end, we have applied our theory to the deformation of low-cohesion materials such as porous sandstones and predict behaviour very similar to that observed in the laboratory.

\section{ACKNOWLEDGMENTS}

Support was provided by NSF (grant EAR-0105269), the Centre National de la Recherche Scientifique (CNRS) and Le Garon Foundation for Supine Geophysics. 


\section{R E F E R E N C ES}

Baud, P., Schubnel, A. \& Wong, T., 2000. Dilatancy, compaction, and failure mode in solnhofen limestone, J. geophys. Res., 105, 19289 19303.

Bercovici, D. \& Ricard, Y., 2003. Energetics of a two-phase model of lithospheric damage, shear localization and plate-boundary formation, Geophys. J. Int., 152, 581-596.

Bercovici, D., Ricard, Y. \& Richards, M., 2000. The relation between mantle dynamics and plate tectonics: a primer, in History and Dynamics of Global Plate Motions, Geophys. Monogr. Ser., Vol. 121, pp. 5-46, eds Richards, M.A., Gordon, R., \& van der Hilst, R., AGU, Washington, DC.

Bercovici, D., Ricard, Y. \& Schubert, G., 2001a. A two-phase model of compaction and damage, 1. General theory, J. geophys. Res., 106, 88878906.

Bercovici, D., Ricard, Y. \& Schubert, G., 2001b. A two-phase model of compaction and damage, 3. Applications to shear localization and plate boundary formation, J. geophys. Res., 106, 8925-8940.

Drew, D., 1971. Averaged field equations for two-phase media, Stud. Appl. Math., 50, 133-166.

Fung, Y., 1977. A First Course in Continuum Mechanics, Prentice-Hall, Englewood Cliffs.

Griffith, A., 1921. The phenomenon of rupture and flow in solids, Phil. Trans. R. Soc. Lond., A, 221, 163-198.

Jaeger, J. \& Cook, N., 1979. Fundamentals of Rock Mechanics, 3rd edn, Chapman and Hall, New York.

Jamison, W. \& Teufel, L., 1979. Pore volume changes associated with failure and frictional sliding of a porous sandstone, Proc. US Rock Mech. Symp, 20, 163-170.

Jizba, D., 1991. Mechanical and acoustical properties of sandstones and shales, PhD thesis, Stanford University.

Khan, A., Xiang, Y. \& Huang, S., 1991. Behavior of berea sanstone under confining pressure, I. Yield and failure surfaces, and nonlinear elastic response, Int. J. Plasticity, 7, 607-624.

Lyakhovsky, V., Ben-Zion, Y. \& Agnon, A., 1997. Distributed damage, faulting, and friction, J. geophys. Res., 102, 27 635-27 649.

McKenzie, D., 1984. The generation and compaction of partially molten rock, J. Petrol., 25, 713-765.

Murrell, S., 1963. A criterion for brittle fracture of rocks and concrete under triaxial stress and the effect of pore pressure on the criterion, in Proc. 5th Rock Mechanics Symp., pp. 563-577, ed. Fairhurst, C., Pergamon, Oxford.

Ni, J. \& Beckerman, C., 1991. A volume-averaged two-phase model for transport phenomena during solidification, Metall. Trans. B, 22, 349361.

Ricard, Y., Bercovici, D. \& Schubert, G., 2001. A two-phase model of compaction and damage, 2, applications to compaction, deformation, and the role of interfacial surface tension, J. geophys. Res., 106, 8907-8924.

Schock, R., Heard, H. \& Stephens, D., 1973. Stress-strain behavior of a granodiorite and two graywackes on compression to 20 kilobars, J. geophys. Res., 78, 5922-5941

Sleep, N., 2002. Self-organization of crustal faulting and tectonics, Int. Geol. Rev., 44, 83-96.

Vernik, L., Bruno M. \& Bovberg, C., 1993. Empirical relations between compressive strength and porosity of siliclastic rocks, Int. J. Rock Mech. Min. Sci. \& Geomech. Abstr., 30, 677-680.

von Mises, R., 1913. Mechanik der festen korper im plastich deformablen zustand, Nachr. Ges. Wiss. Gottingen, pp. 582-592.

Wong, T.-F., David, C. \& Zhu, W., 1997. The transition from brittle faulting to cataclastic flow in porous sandstones: mechanical deformation, J. geophys. Res., 102, 3009-3025.

Zhang, J., Wong, T.-F. \& Davis, D., 1990. Micromechanics of pressureinduced grain crushing in porous rocks, J. geophys. Res., 95, 341-352.

Zhu, W. \& Wong, T.-F., 1997. The transition from brittle faulting to cataclastic flow: permeability evolution, J. geophys. Res., 102, 3027-3041. 